\title{
Insights into the Pulsational Behaviour of $\delta$ Sct Stars
}

\section{Mantegazza}

Università di Pavia, Dipartimento di Fisica Nucleare e Teorica, Via Bassi 6, 27100 Pavia, Italy

E. Poretti

Osservatorio di Brera, Via E. Bianchi 46, 22055 Merate, Italy

\section{Introduction}

Extensive photometric monitoring of bright $\delta$ Scuti stars, made in the last years, allowed us to get reliable frequency determinations also in very complicated cases. Table 1 gives a full description of our photometric targets. Recently, we realized that combining photometry with simultaneous high resolution spectroscopy, in order to study line profile variations, offers the possibility to perform a much more reliable mode identification. Hence, the photometric runs of FG Vir, X Cae, HD 2724 were paired with spectroscopic ones obtained at the Coudé Auxiliary Telescope (ESO, La Silla, Chile). The stellar spectra are centred at $4508 \AA$ and they cover a range of $37.6 \AA$; the resolving power is about 50000 60000 and the linear dispersion is $2.4 \AA / \mathrm{mm}$.

\section{New results}

$X$ Caeli - Already observed in the $V$ colour in $1989, \mathrm{X}$ Caeli was reobserved both phometrically and spectroscopically in 1992 . The frequency analysis of the new photometric data confirmed the presence of a dominant mode (7.39 $\mathrm{c} / \mathrm{d}$, amplitude $37 \mathrm{mmag})$, two modes with intermediate amplitudes $(6.04 \mathrm{c} / \mathrm{d}$, amplitude $7 \mathrm{mmag}$, and $7.46 \mathrm{c} / \mathrm{d}$, amplitude $5 \mathrm{mmag}$ ), many other modes with smaller amplitude, the first Fourier harmonic of the three strongest modes and their nonlinear coupling terms. The amplitude ratios and phase shifts between $V$ and $B-V$ curves suggest that the three strongest modes are nonradial with $\ell=1$ or $\ell=2$.

The availability of light, colour and radial velocity curves allowed us to perform a preliminary application of the Baade-Wesselink method to the dominant mode. There is an acceptable solution for $\ell=2$ only, from which we also obtain a mean stellar radius of $R=5.0 \pm 0.4 R_{\odot}, f=18.9 \pm 1.5$ (ratio of flux to radius variation), $\psi=100.5^{\circ} \pm 3.5^{\circ}$ (phase difference between the radius and the flux variations), $\epsilon=0.0052 \pm 0.0004$ (the projected radial semi-amplitude), $A=2.48 \pm 0.26$ (the gradient of the surface brightness-colour relationship).

In the stellar spectra obtained in 1992, a narrow absorption core is visible at the bottom of the spectral lines. This feature is stable with respect to the stellar reference frame; 9 additional spectra taken in November 1994 confirm 
its stability over an interval of years. Therefore, we could be in presence of a circumstellar shell.

HD 16439 - This star was observed at Merate Observatory in 1988-89. Two frequencies at $17.1266 \mathrm{c} / \mathrm{d}$ and $10.1273 \mathrm{c} / \mathrm{d}$ were clearly identified; no additional peak was visible. HD 16439 was re-observed at Merate Observatory in December 1994 and January 1995 . The presence of the main frequency at $17.13 \mathrm{c} / \mathrm{d}$ was confirmed, while the difference in height between the $10.13 \mathrm{c} / \mathrm{d}$ peak and its alias at $11.13 \mathrm{c} / \mathrm{d}$ is reduced. Moreover, a new peak at $10.48 \mathrm{c} / \mathrm{d}$, not observed in the 1988-89 dataset, is clearly identified in the frequency analysis. Since the two datasets were obtained in the same conditions (site, length of the observations, number of data, ...), the evidence for a change in the frequency content between 1989 and 1994 seems well established. Moreover, the similar height of the peaks at 10.13 and $11.13 \mathrm{c} / \mathrm{d}$ makes one suspect that other undetectable terms are present in this spectral region.

Table 1. Summary of the photometric observations carried out by the Merate group

\begin{tabular}{lccrrl}
\hline \multicolumn{1}{c}{ Star } & Site & Year & N & $\begin{array}{c}\text { Survey } \\
\text { hours] }\end{array}$ & Reference \\
\hline HD 37819 & Merate & 1986 & 462 & 32 & A\&A 1987, 181, 273 \\
HR 1225 & ESO & 1987 & 705 & 38 & A\&A 1989, 220, 144 \\
$o^{1}$ Eri & ESO & 1987 & 710 & 38 & A\&A 1989, 220, 144 \\
HR 547 & ESO & 1987 & 462 & 22 & A\&A 1989, 220, 144 \\
HD 16439 & Merate & $1988-89$ & 1020 & 54 & A\&A 1990, 230, 91 \\
HD 16439 & Merate & $1994-95$ & & & work in progress \\
HD 101158 & ESO & 1989 & 1234 & 62 & A\&A 1991, 245, 136 \\
X Cae & ESO & 1989 & 1013 & 54 & A\&A 1992, 255, 153 \\
X Cae & ESO & 1992 & 1634 & 100 & work in progress \\
44 Tau & Merate & $1989-90$ & 2434 & 117 & A\&A 1992, 256, 113 \\
HD 66853 & ESO & 1991 & 1390 & 100 & A\&A 1994, 281, 66 \\
HD 66853 & Merate & 1991 & 642 & 43 & A\&A 1994, 281, 66 \\
HD 224639 & ESO & 1991 & 2567 & 120 & A\&A 1995, in press \\
HD 224639 & ESO & 1994 & & & work in progress \\
HD 18878 & Merate & $1991-92$ & 2915 & 150 & A\&A 1993, 274, 811 \\
HD 19279 & Merate & $1991-92$ & 2679 & 150 & A\&A 1993, 274, 811 \\
$\beta$ Cas & Merate & 1986 and 1992 & 925 & 30 & A\&AS 1994, 108, 55 \\
FG Vir & ESO & 1992 & 792 & 53 & A\&A 1994, 287, 95 \\
HD 2724 & ESO & 1993 & & & work in progress \\
AZ CMi & Merate & 1994 & & & work in progress \\
\hline
\end{tabular}

